\title{
Here Is the Case for the Urge in Administrative Claims Database Research About Overactive Bladder Therapies
}

\author{
Sheryl L. Szeinbach, PhD, MS, RPh, and Mark Jackson, RPh, BScPhm, BComm
}

W ith an estimated 16.5\% of Americans experiencing symptoms from overactive bladder (OAB) and urinary incontinence (UI), this condition deserves continued research focus. ${ }^{1}$ In this issue of JMCP, D'Souza et al. present a retrospective claims database analysis of adherence, persistence, and switching behaviors, comparing cohorts of patients using different medications to treat OAB. ${ }^{2}$ D'Souza et al. use standard approaches to calculate adherence, persistence, and switching rates. ${ }^{2,3}$ Study patients had at least 1 pharmacy claim for either extended-release (ER) or immediate-release (IR) products for tolterodine or oxybutynin during the period from July 1, 1999, to December 31, 2003. Four patient cohorts (ER vs. IR for each of the 2 drugs) were followed from the index date to either discontinuation (defined as a gap in OAB therapy of at least 45 days), a switch to any other OAB medication, or the end of a 1-year follow-up period. The medication possession ratio was calculated as the sum of the total number of days supplied across all pharmacy claims except the last claim, divided by the total number of days from the first fill date to the last fill date. Persistence was measured as the proportion of patients continuing therapy for 12 months without discontinuation or a switch to another $\mathrm{OAB}$ drug. The switch rate was calculated as the proportion of patients who changed from the initial index medication to any other $O A B$ treatment, including another study drug, a different dosage form of the same drug (e.g., oxybutynin ER to oxybutynin IR), or other medications, including trospium chloride, oxybutynin patch, flavoxate, hyoscyamine sulfate, or propantheline bromide.

D'Souza et al.'s findings pose a challenge to clinicians to find better strategies to diagnose and manage the symptoms of $\mathrm{OAB}$. As revealed in this study, only $55.5 \%$ of patients refilled their first prescription for OAB medication and only $13.2 \%$ persisted with the index medication for 1 year. Also telling is the finding that medication switching appears to be common practice among these patients; the switch rate was $13.3 \%$ for the overall sample and $24.0 \%$ of patients with at least 1 refill. No significant persistence advantages were observed for any of the $4 \mathrm{OAB}$ drug products in the study in multivariate analysis, although the odds of adherence with IR drugs were half that for ER drugs (odds ratio $[\mathrm{OR}]=0.504 ; 95 \% \mathrm{CI}, 0.306-0.704 ; P<0.001)$.

In any study, an assessment of whether the data are appropriate for the task at hand is necessary. The finding that only 53.7\% of the patients had at least $1 \mathrm{OAB}$ diagnosis recorded during the 18-month eligibility period provides evidence that OAB diagnostic information obtained from large databases is not without shortcoming. These low rates for OAB diagnosis pose serious threats to study validity in that D'Souza et al. may have assessed patients with $\mathrm{OAB}$, as well as patients with interstitial cystitis or other UI problems triggered by urinary tract infections. In addition, subjective measures of well being and patient satisfaction, although not reported in claims databases, may also account for patients' predilection to discontinue medications or use them intermittently according to lifestyle. For example, when medications for $\mathrm{OAB}$ are used only during travel, special events, or other activities to minimize the impact of side effects on quality of life, what appears to be discontinuation or non-adherence based on days supply intervals might actually represent a planned pattern of use.

The $13.2 \%$ persistence rate for the use of these drugs for $O A B$ found by D'Souza et al. at 1 year is extremely low compared with persistence rates for other drug therapy classes. In a study conducted using a similar methodology from 1998 to 2000 in Quebec, 1-year persistence rates for angiotensin-converting enzyme inhibitors in cardiovascular disease ranged from 64\% to $72 \%{ }^{4}$ Similarly, a cross-national study of persistence for antihypertensive medication use in the elderly revealed that approximately $25 \%$ of the patients were without medication for at least 180 days during the first year after the initiation of treatment. ${ }^{5}$ After 6 years, these percentages increased to $41.1 \%, 36.3 \%$, and $38.2 \%$ for patients in Pennsylvania, British Columbia, and the Netherlands, respectively. Thus, persistence rates may suggest but do not necessarily explain the rationale for discontinuing or changing drug therapy. Other factors, such as behavioral modification, dietary change, and exercise, though not reported in databases, could improve patient symptoms, in part explaining the high rate of non-persistence. ${ }^{6}$

The statistical techniques employed in the study by D'Souza et al. might have been inadequate to assess intermittent drug use that appears to occur with this particular drug class. In addition to controlling for covariates, such as prescription coverage and comorbidities, intermittent, or cyclic medication use, requires special consideration in data analysis, where censoring (failing to complete treatment) and changes in diagnosis, treatment, and eligibility status complicate analyses. ${ }^{7}$ Analogous to employment and unemployment fluctuations that were originally modeled as duration data, ${ }^{8}$ similar cycles with $\mathrm{OAB}$ medication use may be captured more accurately with an interval-censoring modeling approach to account for variations in medication-taking behavior. In addition, sensitivity analyses, expanding the study grace period of 45 days to include a range from 30 to 90 days may have detected more subtle nuances in medication use.

These questions about persistence and adherence become all the more important as newer agents for the management of $\mathrm{OAB}$ 
are introduced in the market. Managed care professionals are compelled to review both new and old $\mathrm{OAB}$ treatments in the formulary decision-making process.

In 2002, the Canadian Agency for Drugs and Technologies in Health, in conjunction with most publicly funded Canadian provincial drug plans, began developing a process known as the Common Drug Review (CDR). The CDR examines clinical and cost effectiveness of new drugs introduced in Canada to provide formulary recommendations to most provincial drug plans. ${ }^{9}$ The CDR is fundamentally similar to the National Institute for Health and Clinical Excellence (NICE) in the United Kingdom. Both evaluate pharmaceuticals with respect to clinical and cost effectiveness. However, the CDR's mandate is limited to the review of all new chemical entities and combination products, whereas NICE undertakes reviews upon request. . $^{10,11}$

To date, the CDR review for solifenacin found "insufficient evidence that solifenacin provides clinically important differences in outcomes compared with less expensive alternatives." ${ }^{12}$ Reviews for both darifenacin and trospium chloride observed that while theoretical advantages may exist for these agents (i.e., reduced penetration of the blood-brain barrier leading to fewer central nervous system effects), these apparent advantages had not yet been supported through clinical trials in the elderly. ${ }^{13,14}$

These somewhat vague Canadian conclusions had been previously reported by the Oregon Health Resources Commission in a January 2006 report. In this report, the authors agreed not only that evidence of consistent differences in drug efficacy (as measured variously by micturation frequency, number of urgency or incontinence episodes, etc.) was lacking, but that available evidence did not show consistent differences in the incidence of adverse events of drug withdrawals. ${ }^{15}$

The CDR has also expressed concern regarding the growing number of agents to treat $\mathrm{OAB}$, the increased use of these agents, and their risk/benefit profile, urging a class review of the safety, effectiveness, and cost effectiveness of these agents. ${ }^{12,13,14}$ Unfortunately, such a review would fall outside the existing mandate of the CDR as it would involve existing chemical entities, such as oxybutynin and tolterodine. To date, it is unclear how, when, or by whom such a review will be conducted.

The Ontario Ministry of Health and Long-Term Care's Committee to Evaluate Drugs echoed the concerns of the CDR when reviewing trospium chloride and darifenacin, noting that "given the prevalence of the inappropriate use of drugs to treat $\mathrm{OAB}$ and the significant risk of clinically important side effects, especially in the geriatric population (i.e. delirium), the Committee indicated that expanding the use of this class of drugs could negatively effect [sic] the overall health of the Ontario population." ${ }^{16,17}$

As the number of drugs used to treat $\mathrm{OAB}$ has grown, so has the confusion over where newer drugs fit into the treatment of OAB. Unfortunately, the literature is lacking in "real-world" adherence and persistency data on the newer agents. While praising the potential benefits of trospium chloride, Halaska et al. (2003) also noted that the discontinuation rates in their study group were nearly identical between those receiving trospium chloride $(25.0 \%)$ and those receiving oxybutynin (26.7\%). While this study rightfully notes that the relative risk of experiencing an adverse effect favors trospium chloride, the similarity of the overall discontinuation rates raises questions with respect to the clinical significance of the favorable adverse effect profile of the drug. ${ }^{18}$ Hegde (2006) also noted that poor persistence will limit the long-term effectiveness of all available drugs to treat $\mathrm{OAB} \cdot{ }^{19}$

There is clearly a need for a better understanding of adherence, persistence, and switching behavior in those taking drugs to treat $O A B$. Without such understanding, lifestyle preferences and psychological factors will continue to interfere with the long-term benefit of OAB treatments. Research databases contain a wealth of real-world information about general trends in medicationtaking behavior including adherence, persistence, and switching in large populations. However, administrative claim databases fail to account for psychological factors underlying the decision to seek treatment and generally cannot consider other factors, such as incidence and severity of side effects, insurance coverage changes, alternative therapies, and lifestyle needs that may influence adherence and persistence. A concerted effort is needed to link these large databases with other sources of clinical information including patient history, clinical evaluation, and patient surveys.

\section{Authors}

SHERYL L. SZEINBACH, PhD, MS, RPh, is Professor, Division of Pharmacy Practice \& Administration, College of Pharmacy, 500 W 12th Ave., Ohio State University, Columbus, OH 43210. Tel.: 614.688.4249; E-mail: Szeinbach.1@osu.edu

MARK JACKSON, RPh, BScPhm, BComm, is Provider and Professional Services Liaison, Green Shield Canada, 8677 Anchor Dr., P.O. Box 1606, Windsor, Ontario N9A 6W1 Canada. Tel.: 519.739.1133 x 6215; E-mail: Mark.jackson@greenshield.ca

\section{REFERENCES}

1. Stewart WF, Van Rooyen JB, Cundiff GW, Abrams P, Herzog AR, Correy R, et al: Prevalence and burden of overactive bladder in the United States. World J Urol. 2003;20(6):327-36.

2. D'Souza AO, Smith MJ, Miller LA, Doyle J, Ariely R. Persistence, adherence, and switch rates among extended release and immediate release over-active bladder $(\mathrm{OAB})$ medications in a regional managed care plan. J Manag Care Pharm. 2008;14(3):291-301.

3. Andrade SE, Kahler KH, Frech F, Chan AK. Methods for evaluation of medication adherence and persistence using automated databases. Pharmacoepidemiol Drug Saf. 2006;15(8):565-74. 
4. Gogovor A, Dragomir A, Savoie M, Perreault S. Comparison of persistence rates with angiotensin-converting enzyme inhibitors used in secondary and primary prevention of cardiovascular disease. Value Health. 2007; 10(5):431-41.

5. Van Wijk BL, Shrank WH, Klungel OH, Schneeweiss S, Brookhart MA, Avorn J. A cross-national study of the persistence of antihypertensive medication use in the elderly. J Hypertens. 2008;26(1):145-53.

6. Kafri R, Shames J, Raz M, Katz-Leurer M. Rehabilitation versus drug therapy for urge urinary incontinence: long term outcomes. Int Urogynecol J Pelvic Floor Dysfunct. 2008;19(1):47-52.

7. Crystal S, Akincigil A, Bilder S, Walkup JT. Studying prescription drug use and outcomes with Medicaid claims data. Med Care. 2007; 45(10 Suppl 2):S58-S65.

8. Kiefer NM. Economic duration data and hazard functions. J Econ Lit. 1988;26(2):646-79.

9. Canadian Agency for Drugs and Technologies in Health. Common Drug Review. Available at http://cadth.ca/index.php/en/cdr. Accessed February 23, 2008.

10. McMahon M, Morgan S, Mitton C. The Common Drug Review: A NICE start for Canada? Health Policy. 2006 Aug;77(3):339-51.

11. Lee KM, la Fleur DP. Response to "The Common Drug Review: A NICE start for Canada?" Health Policy. 2007 Jan;80(1):1.Epub 2006 April 4.

12. Canadian Agency for Drugs and Technologies in Health. CEDAC Final recommendation on reconsideration and reasons for recommendation: solifenacin. January 24, 2007. Available at http://cadth.ca/media/cdr/ complete/cdr_complete_Vesicare_Jan-24-2007.pdf. Accessed February 23, 2007.
13. Canadian Agency for Drugs and Technologies in Health. CEDAC Final recommendation and reasons for recommendation: trospium chloride. August 24, 2006. Available at http://cadth.ca/media/cdr/complete/cdr_ complete_Trosec_August24-06.pdf. Accessed February 23, 2008.

14. Canadian Agency for Drugs and Technologies in Health. CEDAC Final recommendation and reasons for recommendation: darifenacin. October 19, 2006. Available at http://cadth.ca/media/cdr/complete/cdr_complete_ Enablex_Oct-19-06.pdf. Accessed February 23, 2008.

15. Oregon Health Resources Commission. Agents for overactive bladdersubcommittee report. Update \#3, January 2006. Available at www.oregon. gov/OHPPR/HRC/docs/HRC_Reports/OAB_HRC.pdf. Accessed March 26, 2007.

16. Committee to Evaluate Drugs (CED). Recommendations and Reasons Trospium. Ontario Ministry of Health and Long Term Care, July 2007. Available at www.health.gov.on.ca/english/providers/program/drugs/ced/ pdf/trospium.pdf. Accessed March 25, 2008.

17. Committee to Evaluate Drugs (CED). Recommendations and Reasons Darifenacin. Ontario Ministry of Health and Long Term Care, July 2007. Available at www.health.gov.on.ca/english/providers/program/drugs/ced/ pdf/darifenacin.pdf. Accessed March 25, 2008.

18. Halaska M, Ralph G, Wiedemann A, Primus G, Ballering-Brühl B, Höfner K, Jonas U. Controlled, double-blind, multicentre clinical trial to investigate long-term tolerability and efficacy of trospium chloride in patients with detrusor instability. World J Urol. 2003;20(6):392-99.

19. Hegde SS. Muscarinic receptors in the bladder: from basic research to therapeutics. Br J Pharmacol. 2006 Feb;147(Suppl 2):S80-S87. 